\title{
Assessment of p53 and ATM functionality in chronic lymphocytic leukemia by multiplex ligation-dependent probe amplification
}

\author{
GD te Raa ${ }^{1,2}$, PD Moerland ${ }^{3}$, AC Leeksma ${ }^{1,2}$, IA Derks ${ }^{2}$, H Yigittop ${ }^{4}$, N Laddach $^{4}$, M Loden-van Straaten $^{4}$, V Navrkalova ${ }^{5}$, M Trbusek ${ }^{5}$, \\ DM Luijks ${ }^{1,2}$, T Zenz ${ }^{6}$, A Skowronska ${ }^{7}, M_{\text {Hoogendoorn }}^{8}$, T Stankovic ${ }^{7}$, MH van Oers ${ }^{1,9}$, E Eldering ${ }^{2,9,10}$ and AP Kater ${ }^{\star, 1,2,9,10}$
}

The ATM-p53 DNA-damage response (DDR) pathway has a crucial role in chemoresistance in CLL, as indicated by the adverse prognostic impact of genetic aberrations of TP53 and ATM. Identifying and distinguishing TP53 and ATM functional defects has become relevant as epigenetic and posttranscriptional dysregulation of the ATM/p53 axis is increasingly being recognized as the underlying cause of chemoresistance. Also, specific treatments sensitizing TP53- or ATM-deficient CLL cells are emerging. We therefore developed a new ATM-p53 functional assay with the aim to (i) identify and (ii) distinguish abnormalities of TP53 versus $A T M$ and (iii) enable the identification of additional defects in the ATM-p53 pathway. Reversed transcriptase multiplex ligationdependent probe amplification (RT-MLPA) was used to measure ATM and/or p53-dependent genes at the RNA level following DNA damage using irradiation. Here, we showed that this assay is able to identify and distinguish three subgroups of CLL tumors (i.e., TP53-defective, ATM-defective and WT) and is also able to detect additional samples with a defective DDR, without molecular aberrations in TP53 and/or ATM. These findings make the ATM-p53 RT-MLPA functional assay a promising prognostic tool for predicting treatment responses in CLL.

Cell Death and Disease (2015) 6, e1852; doi:10.1038/cddis.2015.223; published online 6 August 2015

Chronic lymphocytic leukemia (CLL), the most common leukemia in the Western world, is characterized by an extremely variable clinical course. Some patients have stable disease for many years, whereas others rapidly progress with immediate need for chemotherapy. Thus far, cytogenetic aberrations of the pivotal regulators of the DNA-damage response (DDR) pathway, TP53 and ATM, have been shown to provide the most powerful predictive information on clinical outcome and responsiveness to chemotherapy..$^{1-5}$

Approximately $8 \%$ and $18 \%$ of CLL patients requiring frontline therapy harbor defects of TP53 or ATM, respectively. ${ }^{3,6}$ These frequencies increase when the disease progresses following initial therapies. Although TP53 and ATM aberrations both lead to $\mathrm{p} 53$ dysfunction, there are substantial differences both at the clinical and at the cellular level that distinguish TP53-defective from ATM-defective CLL. Clinically, TP53-defective CLL is more aggressive, whereas ATM-defective CLL has a more prolonged clinical course. ${ }^{4}$
At the cellular level, TP53-disruptive CLL exhibits a complete absence of DNA-damage-induced apoptosis in vitro, whereas ATM-disruptive CLL retains a capacity for apoptosis after in vitro-induced DNA damage, though at a reduced level. ${ }^{7,8}$ In addition, microarray analyses revealed that TP53- and ATM-mutant CLL share a defect in activating proapoptotic responses after DNA damage but are distinguished by major differences in activating prosurvival responses. ${ }^{9}$

In CLL, the majority of TP53 defects consists of biallelic TP53 defects (70\%), that is, a TP53 deletion (17p deletion) in one allele in conjunction with a TP53 mutation in the other allele. ${ }^{5}$ In marked contrast, less than $40 \%$ of $A T M$ deletions (11q deletion) coincide with an ATM mutation. ${ }^{2}$ Whereas monoallelic lesions of TP53 (i.e., mutations or deletions) commonly lead to p53 dysfunction and impaired responses to chemotherapy, ${ }^{5,10}$ only biallelic defects of ATM (i.e., mutation and deletion) usually result in impaired p53 response..$^{2,4,11}$

\footnotetext{
${ }^{1}$ Department of Hematology, Academic Medical Center, Amsterdam, The Netherlands; ${ }^{2}$ Laboratory of Experimental Immunology, Academic Medical Center, Amsterdam, The Netherlands; ${ }^{3}$ Bioinformatics Laboratory, Department of Clinical Epidemiology, Biostatistics and Bioinformatics, Academic Medical Center, Amsterdam, The Netherlands; ${ }^{4}$ MRC-Holland, Amsterdam, The Netherlands; ${ }^{5}$ Department of Molecular Medicine, Central European Institute of Technology, Masaryk University, Brno, Czech Republic; ${ }^{6}$ Department of Translational Oncology, National Center for Tumor Diseases (NCT), German Cancer Research Center (DKFZ) and Department of Medicine V, University Hospital Heidelberg, Heidelberg, Germany; ${ }^{7}$ School of Cancer Sciences, University of Birmingham, Birmingham, UK; ${ }^{8}$ Department of Hematology, Medical Center Leeuwarden, Leeuwarden, The Netherlands and ${ }^{9}$ LYMMCARE (Lymphoma and Myeloma Center), Amsterdam, The Netherlands

${ }^{*}$ Corresponding author: AP Kater, Department of Hematology or Laboratory of Experimental Immunology, Academic Medical Center University of Amsterdam, Meibergdreef 9 , Amsterdam, 1105 AZ, The Netherlands. Tel: +31 20 5665785; Fax: +31 20 6919743; E-mail: a.p.kater@amc.nl

${ }^{10}$ Co-senior authors.

Abbreviations: CLL, chronic lymphocytic leukemia; TP53, tumor protein p53; ATM, ataxia telangiectasia mutated; RT-MLPA, reversed transcriptase-multiplex ligationdependent probe amplification; WT, wild type; DNA, deoxyribonucleic acid; DDR, DNA-damage response; FISH, fluorescence in situ hybridization; CDKN1A, cyclindependent kinase N1A; BBC3, bcl-2 homology domain 3; HOVON, Stichting Hemato-Oncologie voor Volwassenen Nederland; iwCLL-NCl, International Workshop on Chronic Lymphocytic Leukemia - National Cancer Institute; DIOC6, 3,3-dihexyloxacarbocyanine iodide; PI, propidium iodide; RNA, ribonucleic acid; IR, ionizing irradiation; PCNA, proliferating cell nuclear antigen; FDXR, ferredoxin reductase; NME1, nm23-H1 gene; PYCR1, pyrroline-5-carboxylate reductase 1; ACSM3, acyl-CoA synthetase medium-chain family member 3; MDM2, mouse double minute 2 homolog
}

Received 12.1.2015; revised 24.6.2015; accepted 01.7.2015; Edited by U Moll 
Currently, detection of deletions via fluorescent in situ hybridization (FISH) of TP53 and ATM is part of standardized clinical work-up in CLL. Analyses of mutations in TP53 and ATM, although of additional clinical value, ${ }^{11,12}$ are currently not standardized and challenging, especially for ATM, owing to its extreme gene size with lack of well-characterized mutations. ${ }^{11,13}$ Particularly, not all sequence variants in ATM lead to pathogenic changes. ${ }^{13}$

In addition to TP53 and ATM defects, chemoresistance might be a consequence of epigenetic and posttranscriptional factors or deregulations of other components of the DDR, because more than $50 \%$ of chemo-refractory CLL patients do not exhibit TP53 or ATM aberrations. ${ }^{5}$

Therefore, functional read-outs of the ATM/p53 axis with the aim to screen for (i) TP53 and ATM mutations, (ii) discrimination between TP53 and ATM defects, and (iii) additional defects in the DDR resulting from mechanisms other than TP53/ATM mutation/deletion, might add clinically relevant information on the actual DDR and chemosensitivity. This type of functional determination could add substantial information to FISH analysis. It is clinically important to distinguish TP53 from ATM defects, because specific treatments that selectively sensitize ATM-deficient tumor cells to killing are emerging. ${ }^{14}$ Previously, we showed that a reverse transcriptase multiplex ligation-dependent probe amplification (RT-MLPA) procedure that quantifies the expression levels of the p53 targets, $C D K N 1 A, B B C 3$ and Bax, in CLL cells following irradiation is able to determine p53 functionality. ${ }^{15}$ With the aim of identifying and distinguishing abnormalities of TP53 versus ATM and enabling the identification of additional defects in the DDR, we developed a new RT-MLPA-based functional assay.

\section{Results}

Prediction of ATM/p53 mutational status using RTMLPA. The RT-MLPA assay was performed on all $(n=30)$ samples from the training cohort and showed upregulation of cluster I genes following ionizing irradiation (IR) in WT samples and impaired upregulation in TP53/ATM-defective CLL samples following IR, confirming earlier results from Stankovic et al. ${ }^{9}$ Additionally, most cluster II-IV genes discriminated between TP53- and ATM-defective CLL samples in their regulation following IR. Results for each probe are shown in Supplementary Figure 2 in terms of fold induction (Fl; expression upon IR in comparison with non-IR). No differences were observed in the expression of any of the included genes in the absence of IR between the different CLL mutational subgroups (data not shown). Because our aim was to develop a highly accurate RT-MLPA assay, in further analyses, we selected those gene probes that robustly discriminated the mutational subgroups (i.e., WT versus TP53/ATM-mutated samples for cluster I genes and TP53defective versus ATM-defective CLL for cluster II-IV genes). Probes were selected by level of significance that resulted from the comparison between the two respective groups. In case of identical $P$-values, probes with the largest change in $\mathrm{FI}$ factors between the two respective groups were selected (Supplementary Table 4). This resulted in a set of 10 probes, containing the following genes: cluster I genes: FAS, Bax,
BBC3, CDKN1 A, PCNA, FDXR; cluster II genes: NME1; cluster III genes: MYC, PYCR1 and cluster IV genes: ACSM3.

To confirm that the 10-gene panel RT-MLPA could distinguish samples according to their ATM/p53 mutational status, we performed a multidimensional scaling analysis, ${ }^{16}$ a statistical method for exploring similarities or dissimilarities in data. Multidimensional scaling analysis showed clear separation between the WT, TP53-mutated and ATM-mutated cases, indicating that the 10-gene panel captured changes in gene expression associated with mutational status (Figure 1a). Next, based on the FI factors of the 10 selected genes, two support vector machine (SVM) classifiers were constructed to enable the classification of CLL samples into three different types of response, that is, ATM/p53 functional, p53dysfunctional or ATM-dysfunctional. Models were constructed in a nested two-step approach. The first SVM predicts whether a sample is either ATM/p53 functional (F) or ATM/p53 dysfunctional (D) based on the Fls of the cluster I genes. The second SVM predicts whether an ATM/p53 dysfunctional sample is either ATM- or p53-dysfunctional based on the Fls of the cluster I-IV genes (Figure 1b). Internal cross-validation of the training set showed that the SVMs correctly classify ATM/p53-dysfunctional, p53-dysfunctional and ATM-dysfunctional patients. Of the WT patients, 13/14 (93\%) were classified as functional and one as dysfunctional. All ATM-defective cases were correctly classified, whereas one out of nine TP53defective samples was classified as ATM-dysfunctional. Note that these estimates are biased by the fact that for each gene in the panel, the most discriminative probe was selected based on the entire training cohort.

Reproducibility of the RT-MLPA. To test for reproducibility of the RT-MLPA assay, we mixed RNA of CLL cells from all included TP53/ATM WT samples and analyzed this sample repeatedly in each experiment. In total, this sample was analyzed 23 times over a period of 3 years. The geometric mean with $95 \%$ confidence intervals for the Fls of individual genes are shown in Supplementary Table 5, illustrating that the RT-MLPA is highly robust with small $95 \%$ confidence intervals for all genes in the panel. Most importantly, all 23 replicate samples were classified consistently as ATM/p53 functional.

Sensitivity of the RT-MLPA. In order to get an insight into the sensitivity of the functional assay in detecting subclones with TP53 and ATM defects, we mixed varying proportions of RNA from CLL cells from patients with either biallelic TP53 or biallelic ATM defects and a large clone size, with those from a patient with WT TP53 and ATM. The assay was able to detect a functional defect when the defective TP53 and ATM clone compromised around $35 \%$ and $45 \%$ of the sample, respectively (Supplementary Figure 3).

Prediction of ATM/p53 mutational status in validation cohort; biallelic lesions. The classification models were validated on a separate cohort (validation cohort; Supplementary Table 1). First, CLL patients from the validation cohort with clear genotypic characteristics, that is, TP53/ATM WT (WT; $n=27$ ), biallelic TP53 defects $(n=6)$ or 

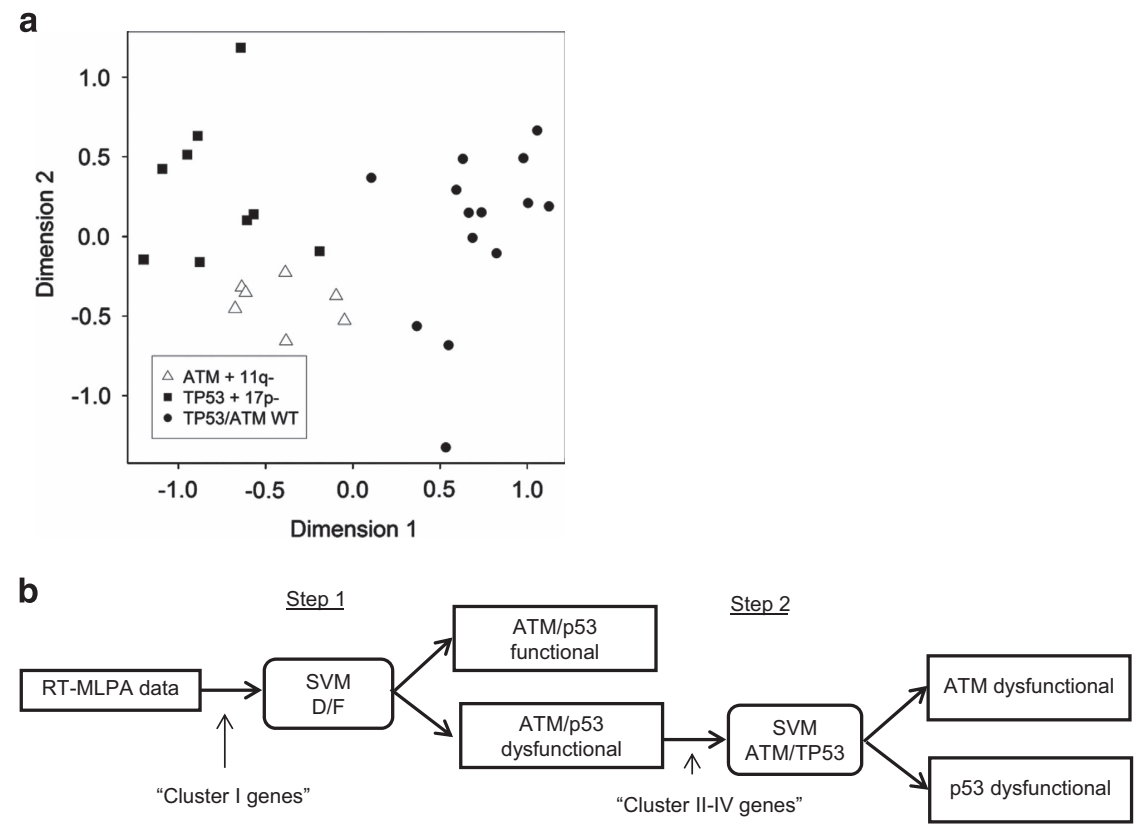

Figure 1 Design of statistical classifier based on the 10-gene panel. (a) Projection of the pairwise relationships among the samples included in the training cohort based on the FI of mRNA expression levels following irradiation and generated using multidimensional scaling analysis (MSA). Each patient is represented by a symbol, with the spatial proximity between any two symbols indicating the degree of similarity between the Fl profiles of 10 selected genes (FAS, Bax, BBC3, CDKN1A, FDXR, PCNA, NME1, MYC, PYCR1, ACSM3) for the two corresponding patients. (b) Schematic overview of the two-stage construction of the support vector machine (SVM) classifiers, which allows classification of CLL samples into three different types of response, that is, ATM/p53-functional, p53-dysfunctional or ATM-dysfunctional based on the Fls of the cluster I-IV genes

biallelic ATM defects ( $n=9)$ (i.e., mutation+deletion) were evaluated. Overall, patterns of response observed in the validation cohort were in agreement with those in the training cohort, with an intact upregulation of cluster I genes in WT and an impaired upregulation in TP53- and ATM-defective samples following irradiation. In addition, TP53-defective cases showed differential expression of cluster II-IV genes following IR in comparison with ATM-defective cases, with upregulation of NME-1, MYC and PYCR1 (cluster II+III) and downregulation of ACMS3. (cluster IV; Figure 2a).

SVM predictions on those samples revealed that all $(6 / 6)$ TP53-defective (17p-+TP53 mutation) samples were classified as p53-dysfunctional. Eight out of nine ATM-defective (11q-+ATM mutation) cases were classified as dysfunctional (i.e., seven ATM-dysfunctional and one p53-dysfunctional) and one ATM-defective sample was classified as functional. Of the WT patients, $21 / 27$ (78\%) were classified as functional, whereas 6/27 (22\%) and 1/27 (3.7\%) were assigned as ATMdysfunctional and p53-dysfunctional, respectively (Figures $2 \mathrm{~b}$ and c). In summary, a high percentage of patients with TP53/ ATM defects were classified as dysfunctional (sensitivity of 93\%), with a high sensitivity for TP53-defects (100\%) and relatively high sensitivity for $A T M$-defects (78\%; Figure $2 \mathrm{c}$ ). In contrast, a relatively high percentage of WT patients were classified as dysfunctional, resulting in a specificity of $78 \%$.

Prediction of ATM/p53 mutational status in samples harboring monoallelic lesions. Next, monoallelic lesions were analyzed, that is, samples with sole TP53 mutation $(n=3)$, sole $17 \mathrm{p}$ deletion $(n=4)$, sole ATM mutation $(n=6)$ and sole $11 \mathrm{q}$ deletion $(n=12)$ (Figures $2 \mathrm{~b}$ and d). All $(3 / 3)$ sole TP53-mutated samples were classified as p53-dysfunctional, whereas one out of four samples with a 17p deletion was classified as p53-dysfunctional (Figure $2 d$ ). The remaining three $17 p$-deleted samples were classified as ATMdysfunctional $(n=1)$ and functional $(n=2)$, respectively. The two samples that were classified as functional harbored the deletion in only 15 and $50 \%$ of the cells, whereas the two samples which were classified as dysfunctional harbored the deletion in 80 and $96 \%$ of cells, suggesting a possible correlation between the degree of p53-(dys)functionality and clone size. With respect to monoallelic ATM aberrations, four out of six sole ATM-mutated cases were classified as ATM-dysfunctional, whereas one case was classified as p53-dysfunctional and one as functional. Nine out of 12 sole $11 q$-deleted samples were classified as functional, whereas 2 displayed an ATM-dysfunctional and one a p53-dysfunctional response. There was no correlation between the clone size of cells harboring an 11q deletion and the degree of ATM-(dys) functionality (data not shown).

In vitro responses to DNA-damaging agents of samples classified according to the RT-MLPA. In addition to TP53 and ATM genetic defects, chemoresistance might be a consequence of other defects in the DDR, especially because more than $50 \%$ of chemo-refractory CLL patients do not exhibit TP53 or ATM aberrations. ${ }^{5}$ Therefore, the RTMLPA could be a very useful tool to detect ATM-p53 dysfunctional patients in the absence of ATM and/or TP53 mutations. Interestingly, six WT samples were classified as dysfunctional (WT+dysf) according to the RT-MLPA-based SVM classifier. To determine whether these samples were 
indeed functionally affected, additional analyses of the in vitro apoptotic response to various DNA-damaging agents, that is, fludarabine, doxorubicin and irradiation were performed. In total, viable cells were available for in vitro testing of four WT+dysf samples. These were compared with WT samples $(n=8)$ that were classified as functional (WT+funct).

a

Cluster I

Apoptosis
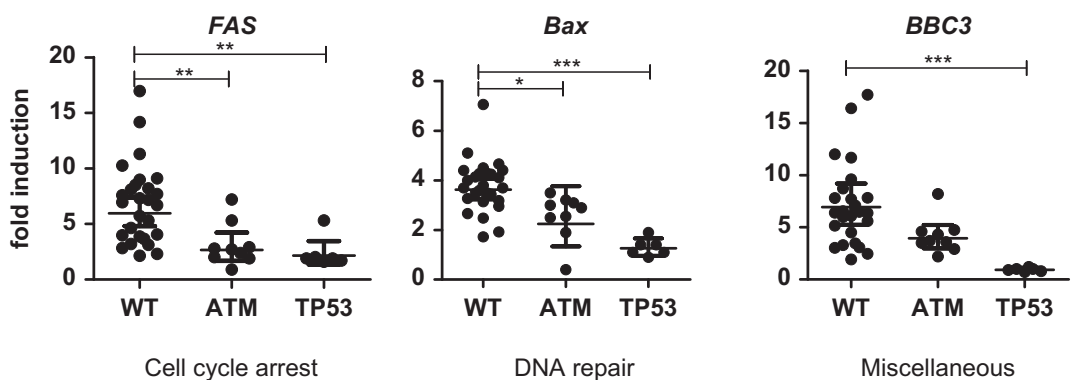

CDKN1A

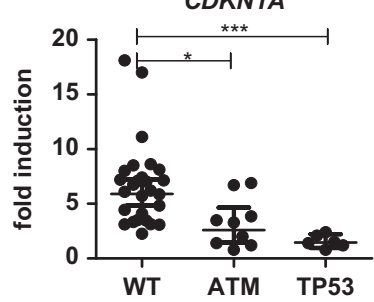

PCNA

FDXR
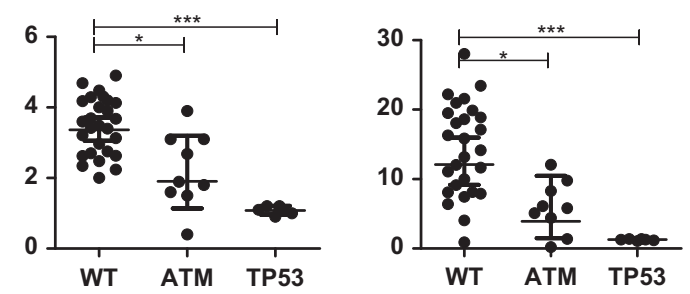

Cluster II

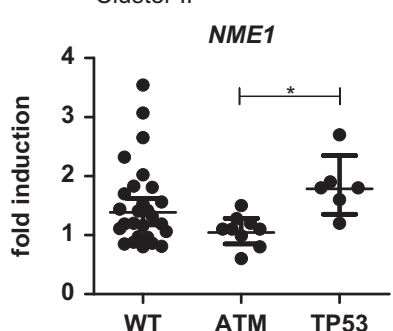

Cluster III
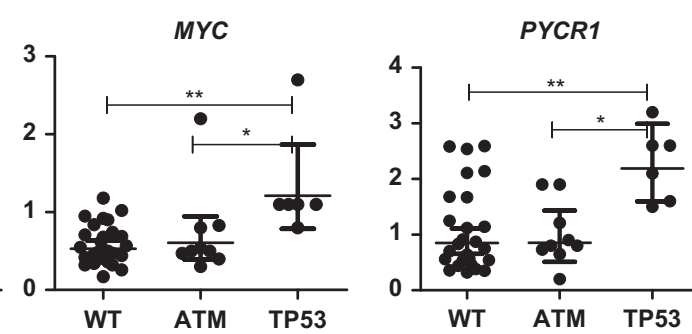

Cluster IV

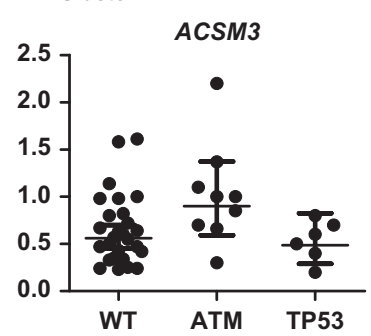

b
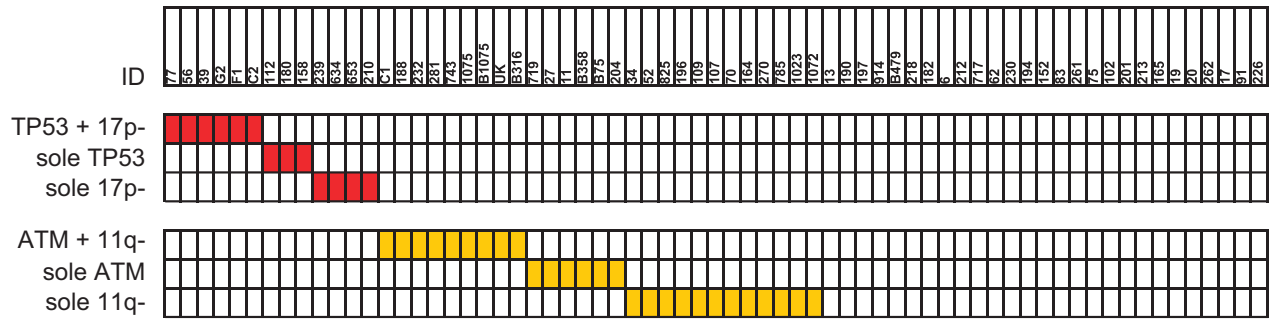

ATM/TP53 W

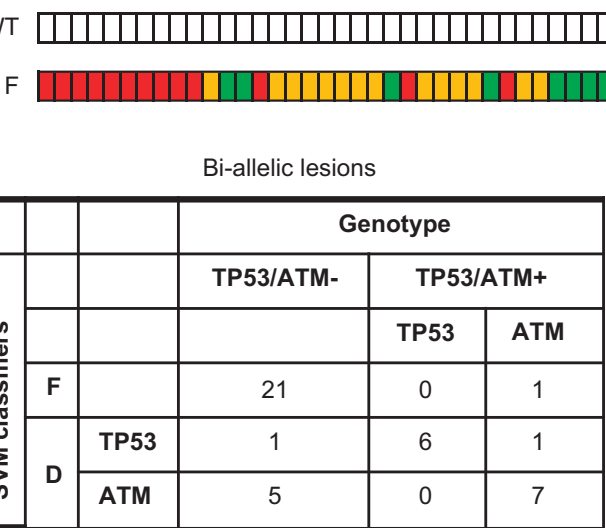

TP53, ATM or F

TP53 aberration present ATM aberration present ATM /TP53 WT

c

\begin{tabular}{|c|c|c|c|c|c|}
\hline & & & \multicolumn{3}{|c|}{ Genotype } \\
\hline \multirow{5}{*}{ 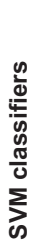 } & & & TP53/ATM- & TP5 & TM+ \\
\hline & & & & TP53 & ATM \\
\hline & $\mathbf{F}$ & & 21 & 0 & 1 \\
\hline & \multirow{2}{*}{ D } & TP53 & 1 & 6 & 1 \\
\hline & & ATM & 5 & 0 & 7 \\
\hline
\end{tabular}

d

\begin{tabular}{|c|c|c|c|c|c|c|}
\hline & & & \multicolumn{4}{|c|}{ Genotype } \\
\hline \multirow{5}{*}{$\begin{array}{l}\frac{n}{\omega} \\
\frac{5}{5} \\
\frac{5}{0} \\
\frac{\pi}{0} \\
\sum_{\infty}^{\infty}\end{array}$} & & & \multicolumn{2}{|c|}{ ATM } & \multicolumn{2}{|c|}{ TP53 } \\
\hline & & & 11q- & $\begin{array}{l}\text { sole } \\
\text { ATM }\end{array}$ & $17 p-$ & $\begin{array}{l}\text { sole } \\
\text { TP53 }\end{array}$ \\
\hline & $\mathbf{F}$ & & 9 & 1 & 2 & 0 \\
\hline & & TP53 & 1 & 1 & 1 & 3 \\
\hline & D & ATM & 2 & 4 & 1 & 0 \\
\hline
\end{tabular}


Interestingly, the WT+dysf samples showed significantly reduced apoptosis to all agents in comparison with the WT +funct samples (Figure 3a), indicating that defects in the DDR other than TP53/ATM aberrations are indeed present and probably responsible for the observed defective DNAdamage-induced apoptotic responses. In addition, apoptotic responses of the sole 11 q-deleted samples, from which viable cells were available, were also examined. This
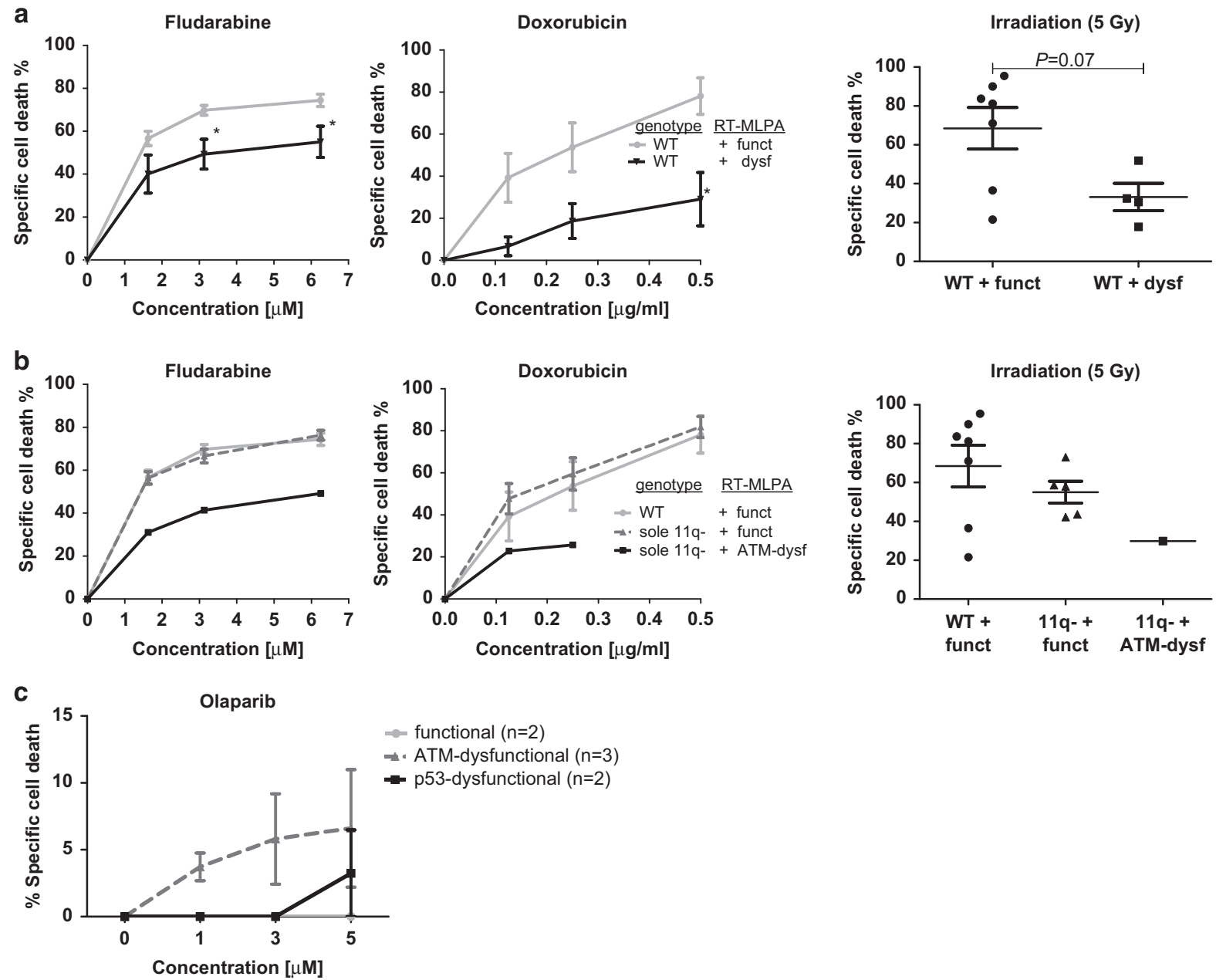

Figure 3 TP53/ATM WT CLL samples that were classified as ATM-dysfunctional display defective apoptotic responses to various DNA-damaging agents. (a, b) CLL cells of eight WT (nos. 6, 160, 647, 534, 854, 812, 912, 532) patients, which were classified as functional, and (a) four WT (nos. 13, 218, 190, 914) patients, which were classified as dysfunctional, and CLL cells of (b) five sole 11q deleted (nos. 785, 1023, 1072, 196, 270) patients, which were classified as p53/ATM-functional, and two sole 11q deleted (nos. 52, 825) patients, which were classified as ATM-dysfunctional, were treated with fludarabine or doxorubicin at increasing concentrations or irradiation (5 Gy). After $48 \mathrm{~h}$, cell death was assessed by $\mathrm{DIOC}_{6} / \mathrm{PI}$ staining and specific cell death was calculated as described in the Materials and Methods section. Presented is mean \pm S.E.M. Significant differences in response compared with functional WT CLL cells at the same concentration are presented as ${ }^{*} 0.01 \leq P<0.05 ;{ }^{* *} 0.001 \leq P<0.01 ;{ }^{* *} P<0.001$ (Mann-Whitney $U$-test). (c) CD40L/II21 activated CLL cells of $n=2$ functional, $n=3$ ATM dysfunctional and $n=2$ p53 dysfunctional CLL samples classified according to the RT-MLPA were treated with olaparib at increasing concentrations. After 3 days, cell death was assessed by $\mathrm{DIOC}_{6} / \mathrm{PI}$ staining and specific cell death was calculated as described in the Materials and Methods section. Presented is mean \pm S.E.M.

Figure 2 Validation of RT-MLPA assay and SVM classifiers in an independent validation cohort. (a) CLL cells of samples included in the validation set with clear genotypic characteristics, that is, TP53/ATMWT ( $n=27)$, biallelic TP53 defects $(n=6)$ or biallelic ATM defects $(n=9)$ (i.e., mutation+deletion) were treated with or without irradiation (5 Gy) followed by measurement of mRNA expression levels using RT-MLPA. FI was calculated as the gene expression following irradiation divided by the gene expression level in the corresponding non-irradiated sample. Symbols represent individual patients. Geometric mean $\pm 95 \% \mathrm{Cl}$ within each group is shown. Significant differences in $\mathrm{Fl}$ are presented as ${ }^{*} 0.01 \leq P<0.05 ;{ }^{* *} 0.001 \leq P<0.01 ;{ }^{* *} P<0.001$ (Kruskal-Wallis test with Dunn's multiple comparison post hoc analysis). (b) Shown are the results of the SVM classifiers on the validation cohort (in lower line). Rows represent TP53 and ATM aberrations, columns represent individual patients. In the upper three rows, color coding is based on: TP53 aberrations (white, absence of TP53 aberration; red, presence of TP53 aberration). In the following three rows, color coding is based on: ATM aberrations (white, absence of ATM aberration; orange, presence of ATM aberration). In the following row, color coding is based on: absence of TP53/ATM aberrations (white, presence of TP53 and/or ATM aberration; green, TP53/ATM WT). In the bottom row, color coding is based on results of SVM classifiers (red, p53-dysfunctional; orange, ATM-dysfunctional; green, p53/ATMfunctional). (c, d) Contingency table for the classification of (c) bi-allelic lesions and (d) mono-allelic lesions 
revealed that the apoptotic responses of the samples that were classified as functional ( $n=5,11 \mathrm{q}$-funct) according to the RT-MLPA-based SVM classifier were normal, whereas responses of the cases that were classified as ATM-dysfunctional ( $n=2,11 q-+$ ATM-dysf) were impaired (Figure $3 \mathrm{~b}$; note that for irradiation only one out of two $11 q-+$ funct samples could be tested). These data indicate that the RT-MLPA assay is able to detect additional defects in the DDR resulting from mechanisms other than TP53/ATM aberrations and can potentially distinguish 11q-deleted samples in a dysfunctional and functional group.

It becomes increasingly important to distinguish TP53 defects from ATM defects, because specific treatments that selectively sensitize ATM-deficient tumor cells to killing, such as the PARP-inhibitor olaparib, are emerging. ${ }^{14}$ To determine whether the RT-MLPA indeed can predict whether CLL cells respond to such specific treatments, cell death of samples classified as functional, ATM-dysfunctional or p53-dysfunctional was measured following olaparib treatment as described. ${ }^{14}$ We observed that olaparib only induced cell death in ATM-dysfunctional CLL cells following $3 \mu \mathrm{M}$ of olaparib (Figure $3 \mathrm{c}$ ), which was in line with the observed levels of cell death as published by Weston et al., ${ }^{14}$ showing that only ATM mutational CLL samples respond to olaparib.

\section{Discussion}

Aberrations that involve the TP53 or ATM gene affect the DDR pathway and are well-known adverse prognostic factors in CLL. Defects of the ATM-p53 pathway can also be caused by other mechanisms, such as polymorphisms in $M D M 2^{17,18}$ and CDKN1A, ${ }^{19}$ hypermethylation of the TP53 promotor $^{20}$ or by novel recurrent mutations, such as those described recently for the SAMHD1 gene. ${ }^{21}$ Identifying and distinguishing TP53 and $A T M$ defects has become increasingly relevant as specific treatments for TP53- and ATM-deficient tumors are emerging. However, mutational analyses of TP53 and ATM in particular are challenging and not yet standardized. The aim of this study was to assess whether functional analysis of the ATM-p53 axis using a newly designed ATM-p53 functional assay could (i) detect TP53 and/or ATM aberrations, (ii) distinguish TP53 defects from ATM defects and (iii) enable the identification of additional defects in the ATM-p53 pathway.

In this study, we developed an RT-MLPA-assay that included genes differentially expressed upon irradiation between (i) WT and TP53/ATM-mutant CLL, and between (ii) TP53-mutant and ATM-mutant CLL. The RT-MLPA assay was subsequently evaluated in a training cohort with CLL samples with known TP53 and ATM status, and support vector machine classifiers were constructed based on the Fls upon irradiation for a 10-gene panel. The RT-MLPA assay and SVM classifiers were validated in a separate validation cohort. CLL samples with clear genotypic characteristics (i.e., biallelic defects) were assigned with a high degree of confidence to one of the three categories with sensitivities of $93 \%, 100 \%$ and $78 \%$ for TP53/ ATM WT, biallelic TP53-defective and biallelic ATM-defective samples, respectively. Thus, the RT-MLPA can both identify and distinguish biallelic TP53 and ATM defects with a high degree of confidence.
Interestingly, a substantial number of WT samples were classified as ATM-dysfunctional (22\%, 6/27), which might be cases that harbor other defects in the ATM-p53 pathway than aberrations of TP53 and ATM. This is corroborated by the fact that apoptotic responses to various DNA-damaging agents were affected in the four cases that were further evaluated. Mutational analysis to uncover an underlying mechanism that could be involved in the observed defective DNA-damageinduced responses showed that two cases carried an SF3B1 mutation, ${ }^{22}$ whereas the underlying defects in the other two samples remains elusive. These cases underscore the clinically highly relevant divergence between determination of ATM/p53 status by functional testing and by mutational analysis.

Samples with mono-allelic defects were not included in the training cohort and results were therefore more ambiguous. Mono-allelic deletions (i.e., sole $17 p$ deletion and sole $11 \mathrm{q}$ deletion) were often classified as functional, whereas monoallelic mutations (i.e., sole TP53 mutation and sole ATM mutation) were often classified as p53-dysfunctional and ATM-dysfunctional, respectively. CLL samples with a sole 17p deletion are likely to be classified as functional by the RT-MLPA owing to a low clone size ( $\pm<40-50 \%)$. This is in agreement with other available p53-function assays. ${ }^{10,23,24}$ Sole 11q-deleted samples were often classified as functional (9/12), however, this was not clone-size dependent, as there was no correlation between the percentage of deleted cells and the degree of ATM-(dys)functionality. It is more likely that these samples were labeled functional owing to redundancy of the ATM-kinase activity in the remaining allele, in line with previous studies showing that CLL samples with both ATM alleles affected (either deletion and mutation or two mutations) lack ATM activity, while patients with monoallelic lesions may have preserved ATM function. ${ }^{1,2}$ Although the number of investigated samples was low, the classification of the sole 11q-deleted samples according to the RT-MLPA indeed seems to be correct, because additional testing of apoptotic responses showed that two sole 11q-deleted samples that were classified as ATM-dysfunctional displayed impaired apoptotic responses, while the samples that were classified as functional showed intact apoptotic responses. Why 3 out of 12 sole 11q-deleted samples are classified as dysfunctional and the remaining ones as functional remains to be elucidated, but could be because of the involvement of currently unknown associated mutations or other factors.

Over the past years, several functional assays have been developed to test p53/ATM functionality, such as MIR34a, RT-PCR_CDKN1A and FACSp53-p21. ${ }^{10,18,24,25}$ The majority of these functional assays were designed to assess p53 functionality and not to detect ATM defects specifically nor to distinguish TP53 defects from ATM defects. In none of these studies, mutational analysis of ATM was performed. Some functional assays have been developed with the aim to identify and distinguish TP53- and ATM-defective tumors. ${ }^{11,26,27}$ One such assay is based on monitoring p53 and p21 accumulation after cell exposure to etoposide and nutlin-3a enabling the differentiation of TP53 and ATM defects using flow cytometry. ${ }^{26}$ An alternative assay, based on measuring CDKN1A levels by RT-PCR following fludarabine and doxorubicin treatment, was primarily designed for ATM function 
testing and can also distinguish between TP53 and ATM defects. ${ }^{11}$ As we have previously published, also cell death following DNA-damaging agents can distinguish a group of functional WT samples from a group of ATM-mutated and a group of TP53-mutated samples; ${ }^{22}$ however, this method seems less suitable to functionally test samples at the individual level to predict (dys)functionality, as exemplified in Figure 3a showing that two out of seven WT samples showed small percentages of cell death following irradiation, comparable with the percentage of cell death seen in dysfunctional patients. Finally, promising results were shown for the detection of ATM defects by measuring the percentage of mitotic cells with p53 localization at the centrosome. ${ }^{27}$ So far, these assays have not been validated in a separate cohort using the cutoff values determined in the initial study population, which is an important component of biomarker development.

A potential limitation of functional testing, not only in our study, but also in other studies evaluating p53 functional assays, is that small clones can be missed. ${ }^{10,23,24}$ This is especially important, because there is emerging evidence that the presence of mutations in subclones or in very small clones impact patient outcome, leading to reduced survival. ${ }^{28,29}$ Another potential limitation is that functional testing usually needs viable cells with high purity. In case the viability of cells is low $(<50 \%)$, the RTMLPA functional assay is not reliable and WT samples could incorrectly be classified as dysfunctional.

In conclusion, the newly designed ATM-p53 RT-MLPA assay is able to distinguish three subgroups of CLL tumors (i.e., TP53-defective, ATM-defective and WT) and was also able to detect additional samples with a functional defective DDR, without molecular defects of TP53 and/or ATM. This indicates that the ATM-p53 RT-MLPA might not only be of additional clinical value over FISH to screen for mutations of TP53 and ATM instead of sequencing, but might also be useful for screening of other defects in the DDR pathway in addition to ATM and/or TP53 aberrations. Whether the newly developed ATM-p53 RT-MLPA assay also predicts for clinical outcome in addition to the molecular status of ATM and/or TP53 needs to be further evaluated in large clinical prospective studies.

\section{Materials and Methods}

Patient and samples. A cohort of $30 \mathrm{CLL}$ patients from the Academic Medical Center, Amsterdam, the Netherlands and from the Central European Institute of Technology (CEITIC), Brno, Czech Republic, was enrolled in this study and utilized to set-up the RT-MLPA functional assay (training cohort). An independent second cohort consisted of $67 \mathrm{CLL}$ patients included in the HOVON68 clinical trial, ${ }^{30}$ which was further enriched for patients with TP53 and ATM aberrations from CEITEC (validation cohort). Clinical and genotypic characteristics are described in Supplementary Table 1. For further details on the training cohort, see also Supplementary Methods and Supplementary Figure 1. The study was conducted in accordance with the Declaration of Helsinki and written informed consent was obtained from all patients. Diagnosis of CLL was assessed according to IWCLL-NCI Working Group criteria. Peripheral blood mononuclear cells were isolated and frozen as described earlier. ${ }^{31}$ After thawing, CLL cells were enriched, in case CD19/CD5 purity was below $90 \%$, via negative depletion using $\alpha$-CD3 (CLB-T3/4,1, $\mathrm{nr} 70,1 \mathrm{x} 1), \quad \alpha$-CD14 (CLB-mon/1,nr143,8G3) and $\alpha$-CD16 (CLB-FCRgran1, nr142,5D2) (CLB, Amsterdam, the Netherlands) as described. ${ }^{31}$ Samples with a cell viability $<50 \%, 16 \mathrm{~h}$ after thawing, determined by 3,3-dihexyloxacarbocyanine iodide (Invitrogen, Carlsbad, CA, USA) and propidium iodide (Sigma-Aldrich, St. Louis, MO, USA) using flow cytometry as described, ${ }^{31}$ were excluded from the analysis.
Molecular analyses of TP53 and ATM. Deletions at the 11q22-q23 (ATM), 17p13 (TP53), 13q14 loci and trisomy of chromosome 12 were detected by FISH by using locus-specific probes (Abott Vysis Inc., Des Plaines, IL, USA or MetaSystems, Altussheim, Germany). TP53 (ex4-10) mutational analysis was performed by next generation sequencing using the GS Junior 454 platform (Roche, Basel, Switzerland) ${ }^{32}$

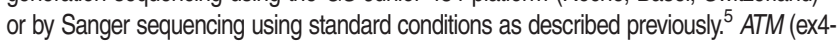
65) was analyzed by either Sanger sequencing and ATM functional analysis assessing irradiation induced phosphorylation of ATM targets or by resequencing microarray and direct sequencing as described previously. ${ }^{111}$

Apoptosis induction by various DNA-damaging agents. Thawed CLL cells were cultured at a concentration of $1.5 \times 10^{6} / \mathrm{ml}$ in the presence of fludarabine or doxorubicin (Sigma-Aldrich) or following exposure to irradiation (5 Gy), for $48 \mathrm{~h}$ at $37^{\circ} \mathrm{C}$. For olaparib studies, thawed CLL cells were stimulated with a CD40/ IL-21 culture system for 4 days. Briefly, murine fibroblast cells (3T3) expressing CD40L were irradiated ( $30 \mathrm{~Gy}$ ) and divided over 48-well plates. After attachment of the fibroblasts, $0.5 \times 10^{6} \mathrm{CLL}$ cells were seeded into each well with $25 \mathrm{ng} / \mathrm{ml} \mathrm{IL-21}$ (Gibco, Carlsbad, CA, USA; Invitrogen) in a total volume of $500 \mu \mathrm{l}$ per well and incubated at $37^{\circ} \mathrm{C}$ for 4 days. Cells were then harvested and replated on CD40Lexpressing $3 \mathrm{~T} 3$ cells and incubated with olaparib (AstraZeneca, London, UK) at various doses in the presence of IL-21 for an additional 3 days. Apoptosis was measured by flow cytometry as previously described. ${ }^{31}$ Specific cell death was calculated as (\%apoptosis $s_{\text {treated cells }}-\%$ apoptosis untreated cells $) \%$ viable untreated cells. $_{\text {. }}$

p53 and ATM target gene induction. CLL cells were treated with or without irradiation ( $5 \mathrm{~Gy}$ ) and cultured for $16 \mathrm{~h}$ at a concentration of $5.0 \times 10^{6}$ cells/ $\mathrm{ml}$ at $37^{\circ} \mathrm{C}$. RNA was isolated using an RNA-isolation kit (Sigma-Aldrich) according to the manufacturer's instructions and subsequently RT-MLPA was performed.

Design of p53/ATM RT-MLPA assay. A new RT-MLPA probe set (R016X2, MRC-Holland), which included several p53 and ATM target genes was designed. Genes were selected based on the results of an earlier microarray study. ${ }^{9}$ In that study, genes differentially expressed between WT $(n=5)$, TP53-mutated $(n=5)$ and ATM-mutated $(n=6) \mathrm{CLL}$ samples in response to DNA-damage using IR were determined and classified into four major clusters. Cluster I represented genes normally upregulated in response to $I R$ in the presence of functionally active ATM and p53, whereas clusters II-IV represented genes whose transcription was upregulated after IR in the presence of functionally inactive p53 (cluster III) or whose transcription was not upregulated or not downregulated in the presence of inactive ATM (cluster II and IV, respectively; Supplementary Table 2). The previous RT-MLPA $\mathrm{kit}^{15}$ contained three cluster I genes and no genes from the other clusters. In the current RT MLPA assay, additional cluster I genes and at least two genes for each of the clusters II-IV were added. See Supplementary Methods for further details on the selection of genes and design of the RT-MLPA kit. For each gene, at least two hemiprobes were designed, which, if possible, span exon boundaries, precluding the detection of potentially contaminating genomic DNA. Furthermore, four housekeeping genes, that is, Diablo, Aif, Gusb and Parn, were included. The housekeeping genes were selected from an earlier RT-MLPA assay design (apoptosis kit R011-C1, MRC-Holland), because their expression was not influenced by irradiation as established by geNorm software. ${ }^{33}$ Target genes and probes are listed in Supplementary Table 3.

Reversed transcriptase multiplex ligation-dependent probe amplification. For preparation of the M13-derived MLPA probe oligonucleotides, reaction conditions and detailed further information on RT-MLPA in general (see Eldering et $\mathrm{al}^{34}$ and MRC Holland website). Expression levels in a sample were normalized to the geometric mean of the expression of the four housekeeping genes in the same sample. For each patient, Fls were calculated by dividing the expression level in the irradiated sample by the expression level in the corresponding non-irradiated sample.

Statistical analysis. A non-parametric Mann-Whitney $U$ test was used for comparison of two independent groups in the training cohort and a non-parametric Kruskal-Wallis test with Dunn's multiple comparison post hoc analysis was used for comparison of multiple groups in the validation cohort. Correlations were analyzed by Spearman's rank correlation test. A $P$-value $<0.05$ was considered statistically significant.

\section{Conflict of Interest}

The authors declare no conflict of interest. 
1. Austen B, Powell JE, Alvi A, Edwards I, Hooper L, Starczynski J et al. Mutations in the ATM gene lead to impaired overall and treatment-free survival that is independent of IGVH mutation status in patients with B-CLL. Blood 2005; 106: 3175-3182.

2. Austen B, Skowronska A, Baker C, Powell JE, Gardiner A, Oscier D et al. Mutation status of the residual ATM allele is an important determinant of the cellular response to chemotherapy and survival in patients with chronic lymphocytic leukemia containing an 11q deletion. J Clin Oncol 2007; 25: 5448-5457.

3. Dohner H, Fischer K, Bentz M, Hansen K, Benner A, Cabot G et al. p53 gene deletion predicts for poor survival and non-response to therapy with purine analogs in chronic B-cell leukemias. Blood 1995; 85: 1580-1589.

4. Skowronska A, Parker A, Ahmed G, Oldreive C, Davis Z, Richards S et al. Biallelic ATM inactivation significantly reduces survival in patients treated on the United Kingdom Leukemia Research Fund Chronic Lymphocytic Leukemia 4 trial. J Clin Oncol 2012; 30: 4524-4532.

5. Zenz T, Eichhorst B, Busch R, Denzel T, Häbe S, Winkler D et al. TP53 mutation and survival in chronic lymphocytic leukemia. J Clin Oncol 2010; 28: 4473-4479.

6. Stankovic T, Weber P, Stewart G, Bedenham T, Murray J, Byrd PJ et al. Inactivation of ataxia telangiectasia mutated gene in B-cell chronic lymphocytic leukaemia. Lancet 1999; 353: 26-29.

7. Pettitt AR, Sherrington PD, Stewart G, Cawley JC, Taylor AM, Stankovic T et al. p53 dysfunction in B-cell chronic lymphocytic leukemia: inactivation of ATM as an alternative to TP53 mutation. Blood 2001; 98: 814-822.

8. Stankovic T, Stewart GS, Fegan C, Biggs P, Last J, Byrd PJ et al. Ataxia telangiectasia mutated-deficient B-cell chronic lymphocytic leukemia occurs in pregerminal center cells and results in defective damage response and unrepaired chromosome damage. Blood 2002; 99: 300-309.

9. Stankovic T, Hubank M, Cronin D, Stewart GS, Fletcher D, Bignell CR et al. Microarray analysis reveals that TP53- and ATM-mutant B-CLLs share a defect in activating proapoptotic responses after DNA damage but are distinghuished by major differences in activating prosurvival responses. Blood 2004; 103: 291-300.

10. Mohr J, Helfrich $H$, Fuge M, Eldering $E$, Bühler A, Winkler D et al. DNA damage-induced transcriptional program in CLL: biological and diagnostic implications for functional p53 testing. Blood 2011; 117: 1622-1632.

11. Navrkalova V, Sebejova L, Zemanova J, Kminkova J, Kubesova B, Malcikova J et al. ATM mutations uniformly lead to ATM dysfunction in chronic lymphocytic leukemia: application of functional test using doxorubicin. Haematologica 2013; 98: 1124-1131.

12. Pospisilova S, Gonzalez D, Malcikova J, Trbusek M, Rossi D, Kater AP et al. ERIC recommendations on TP53 mutation analysis in chronic lymphocytic leukemia. Leukemia 2012; 26: 1458-1461.

13. Stankovic T, Skowronska A. The role of ATM mutations and 11q deletions in disease progression in chronic lymphocytic leukemia. Leuk Lymphoma 2014; 55: 1227-1239.

14. Weston VJ, Oldreive CE, Skowronska A, Oscier DG, Pratt G, Dyer MJ et al. The PARP inhibitor olaparib induces significant killing of ATM-deficient lymphoid tumor cells in vitro and in vivo. Blood 2010; 116: 4578-4587.

15. Mous R, Jaspers A, Luijks DM, Mellink CH, van Oers MH, Kater AP et al. Detection of p53 dysfunction in chronic lymphocytic leukaemia cells through multiplex quantification of p53 target gene induction. Leukemia 2009; 23: 1352-1355.

16. Cox TF, Cox MAA. Multidimensional Scaling. Boca Raton: Chapman \& Hall, 2nd edition. 2011.

17. Zent CS. Time to test CLL p53 function. Blood 2010; 115: 4154-4155.

18. Asslaber D, Pinon JD, Seyfried I, Desch P, Stöcher M, Tinhofer I et al. microRNA-34a expression correlates with MDM2 SNP309 polymorphism and treatment-free survival in chronic lymphocytic leukemia. Blood 2010; 115: 4191-4197.

19. Johnson GG, Sherrington PD, Carter A, Lin K, Liloglou T, Field JK et al. A novel type of p53 pathway dysfunction in chronic lymphocytic leukemia resulting from two interacting single nucleotide polymorphisms within the p21 gene. Cancer Res 2009; 69: 5210-5217.

20. Valganon M, Giraldo P, Agirre X, Larráyoz MJ, Rubio-Martinez A, Rubio-Felix D et al. p53 Aberrations do not predict individual response to fludarabine in patients with B-cell chronic lymphocytic leukaemia in advanced stages Rai III/IV. Br J Haematol 2005; 129: 53-59.

21. Clifford R, Louis T, Robbe P, Ackroyd S, Burns A, Timbs AT et al. SAMHD1 is mutated recurrently in chronic lymphocytic leukemia and is involved in response to DNA damage. Blood 2014; 123: 1021-1031.

22. te Raa GD, Derks IA, Luijks DM, van Laar J, Monsuur H, Oldreive $\mathrm{C}$ et al. SF3B1 mutations in CLL are equivalent to p53/ATM dysfunction and cause defective puma upregulation in response to chemotherapy. Blood (ASH Annual Meeting Abstracts) 8-11 December 2012. Abstract 711.

23. Carter A, Lin K, Sherrington PD, Atherton M, Pearson K, Douglas A et al. Imperfect correlation between p53 dysfunction and deletion of TP53 and ATM in chronic lymphocytic leukaemia. Leukemia 2006; 20: 737-740.

24. Lin K, Adamson J, Johnson GG, Carter A, Oates M, Wade R et al. Functional analysis of the ATM-p53-p21 pathway in the LRF CLL4 trial: blockade at the level of p21 is associated with short response duration. Clin Cancer Res 2012; 18: 4191-4200.

25. Mraz M, Malinova K, Kotaskova J, Pavlova S, Tichy B, Malcikova J et al. miR-34a, miR-29c and miR-17-5p are downregulated in CLL patients with TP53 abnormalities. Leukemia 2009; 23: $1159-1163$.

26. Best OG, Gardiner AC, Majid A, Walewska R, Austen B, Skowronska A et al. A novel functional assay using etoposide plus nutlin-3a detects and distinguishes between ATM and TP53 mutations in CLL. Leukemia 2008; 22: 1456-1459.

27. Prodosmo A, De AA, Nistico C, Gabriele M, Di Rocco G, Monteonofrio $L$ et al. p53 centrosomal localization diagnoses ataxia-telangiectasia homozygotes and heterozygotes. J Clin Invest 2013; 123: 1335-1342.

28. Landau DA, Carter SL, Stojanov P, McKenna A, Stevenson K, Lawrence MS et al. Evolution and impact of subclonal mutations in chronic lymphocytic leukemia. Cell 2013; 152: 714-726.

29. Rossi D, Khiabanian H, Spina V, Ciardullo $\mathrm{C}$, Bruscaggin A, Famà R et al. Clinical impact of small TP53 mutated subclones in chronic lymphocytic leukemia. Blood 2014; 123 : 2139-2147.

30. Geisler $\mathrm{CH}$, van $\mathrm{T}^{\prime}$ Veer $\mathrm{MB}$, Jurlander J, Walewski J, Tjønnfjord G, Itälä Remes $\mathrm{M}$ et al. Frontline low-dose alemtuzumab with fludarabine and cyclophosphamide prolongs progression-free survival in high-risk CLL. Blood 2014; 123: 3255-3262.

31. Mackus WJ, Kater AP, Grummels A, Evers LM, Hooijbrink B, Kramer MH et al. Chronic lymphocytic leukemia cells display p53-dependent drug-induced Puma upregulation. Leukemia 2005; 19: 427-434.

32. Jethwa A, Hullein J, Stolz T, Blume C, Sellner L, Jauch A et al. Targeted resequencing for analysis of clonal composition of recurrent gene mutations in chronic lymphocytic leukaemia. Br J Haematol 2013; 163: 496-500.

33. Vandesompele J, De PK, Pattyn F, Poppe B, Van Roy N, De Paepe A et al. Accurate normalization of real-time quantitative RT-PCR data by geometric averaging of multiple internal control genes. Genome Biol 2002; 3: RESEARCH0034.

34. Eldering E, Spek CA, Aberson HL, Grummels A, Derks IA, de Vos AF et al. Expression profiling via novel multiplex assay allows rapid assessment of gene regulation in defined signalling pathways. Nucleic Acids Res 2003; 31: e153.

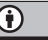

Cell Death and Disease is an open-access journal published by Nature Publishing Group. This work is licensed under a Creative Commons Attribution 4.0 International License. The images or other third party material in this article are included in the article's Creative Commons license, unless indicated otherwise in the credit line; if the material is not included under the Creative Commons license, users will need to obtain permission from the license holder to reproduce the material. To view a copy of this license, visit http://creativecommons.org/licenses/by/4.0/ 